\title{
Concealed deciduous root fragment-cause of recurrent gingival pain: A case report
}

\author{
Nirupama Tayal ${ }^{1}$, Deepak Kochar ${ }^{2, *}$, Shiva Gupta ${ }^{3}$, Rasveen Kaur ${ }^{4}$, Supreet Kaur Thind ${ }^{5}$ \\ ${ }^{1}$ Post Graduate Student, ${ }^{2}$ Professor, ${ }^{3-5}$ Senior Lecturer, Dept. of Periodontology, MM College of Dental Sciences and Research, \\ (Deemed to be University) Ambala, Haryana, India
}

*Corresponding Author:

Email: drdeepakkochar@mmumullana.org

\begin{abstract}
Radiographs are the most useful imaging technique from a clinical perspective when broad coverage of the jaws is needed for diagnosis. Several incidental finding can be seen such as impacted teeth, carotid artery calcification, variants in anatomical landmarks, foreign bodies and retained root fragments. Even though, embedded roots have come across as incidental radiographic findings without any symptoms these roots can cause difficulties in some dental treatments. So here, we report a case of pain and tenderness in lower molar tooth region which was a consequence of retained root fragment.
\end{abstract}

Keywords: Deciduous dentition, Embedded portion, Gingivitis, Retained root fragment, Root resorption, Periodontitis.

\section{Introduction}

Root resorption of deciduous teeth is a physiological process that is a part of normal growth and development of the dentition enabling exfoliation of the deciduous dentition and eruption of successional permanent teeth. The roots of deciduous teeth resorb when the permanent tooth begins active eruption and the follicle comes into close proximity with the root surface. ${ }^{1}$ However, when the roots of deciduous (primary) tooth remains embedded and does not gets resorbed within the mandible or maxilla despite the eruption of the permanent successor, these are known as retained root fragments. The most commonly affected tooth is the deciduous second molar. A retained root is identified by its shape, the associated root canal, the surrounding periodontal ligament space and some evidence of lamina dura. Most commonly seen on mesial or distal of the second premolar. These are found more in mandible than maxilla. ${ }^{2}$ Retained root fragments are common findings appearing as radiolucent landmarks on the radiographs, usually not accompanied by any symptoms or complaints. ${ }^{3}$ However, retained roots also have the propensity to cause pain and discomfort. ${ }^{4}$ Although the literature contains several studies related to the permanent retained root, very limited amount of data were found about embedded primary molar roots in the jaws of adult patients. The aim of the present case report is to evaluate the consequences of retained root fragments followed by the treatment involved.

\section{Case Report}

A twenty-one year old female patient reported to the Department of Periodontology, Maharishi Markandeshwar College of Dental Science and Research, Mullana Ambala, with the chief complaint of constant gingival pain and pressure in right lower back jaw region. The patient also complained of dull pain from last 4 years with bouts of acute pain, cold sensitivity and bleeding gums in the same region. On visual examination, the gingiva was normal with no visual signs of inflammation but on probing the affected area, the periodontal probe was opposed by a hard mass in the interproximal sulcus area of mandibular right second premolar and first molar region. (Fig. 1) A submerged deciduous root fragment was suspected and IOPA was done and root fragment was confirmed. (Fig. 2) Diagnosis of retained root fragment was made on the basis of clinical history and conventional radiograph. The affected area was anaesthetised by local infiltration and with the help of curette, root fragment was removed without any complication. (Fig. 3,4) On follow-up visit after 15 days, uneventful healing was seen in the affected area (Fig. 5) and the patient was relieved from all the concerned symptoms.

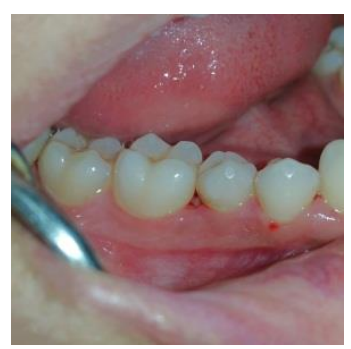

Fig. 1: Pre-operative clinical view

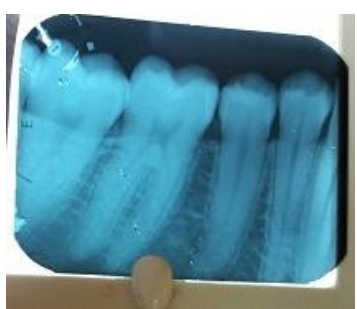

Fig. 2: Pre-operative radiograph 


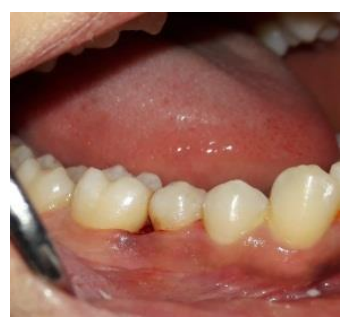

Fig. 3: Inra-operative view

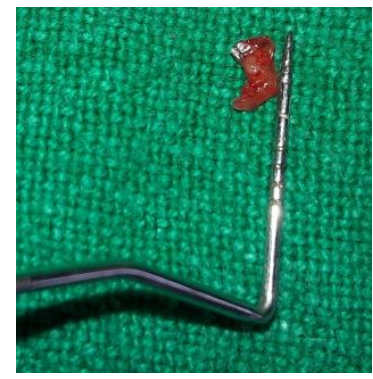

Fig. 4: Retained root fragment

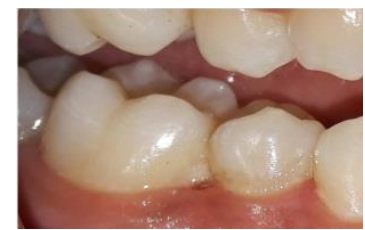

Fig. 5: 15 days post-operative view

\section{Discussion}

In edentulous patients, the prevalance of retained root fragments is reported as $11-37 \%,{ }^{5}$ while in partially dentate mouths prevalance has been reported to have a lower frequency, with incidences of $20 \%,{ }^{2}$ $13 \%{ }^{6}$ and $11 \% .^{7}$ Retained deciduous roots present no problems as long as a healthy attachment apparatus exists in the coronal part of the root fragment. If, however, a periodontal disease process should occur, the deepening sulcus and plaque front would soon encounter the root tip. Considering the problems that could occur, it is important that such root tips be removed when the primary tooth exfoliates. Differential diagnosis of retained roots can be foreign body gingivitis, sequestrum, periapical cemental dysplasia, idiopathic osteosclerosis, ossifying fibroma and condensing osteitis. ${ }^{8}$ The primary retained root fragment diagnosed in the current study have distinguishing features from the foreign body gingivitis and sequestrum, in terms of its location and related clinical signs and symptoms. Embedded root fragments without an associated radiolucent area do not usually require surgical intervention. However, the need for routine radiographic examination is explained to patients and also the possible complications, such as acute situations resulting from the presence of these fragments. If the retained root is associated with a radiolucent area, the root should definitely be removed. If it is not removed, the root may become infected and serious problems could occur. Persistence of root fragments can lead to some clinical problems including gingivitis, periodontitis, profound caries and ankylosis. It can also cause difficulties in some dental treatments, such as orthodontics, fabrication of dentures and dental implants. ${ }^{4,9}$ Nyyssönen et al have proposed that infected retained roots may act as an additional health hazard and that they should be extracted or otherwise treated. When these fragments are studied histologically, it is seen that only fragments presenting with a clinical abnormality, such as pain or a sinus tract, have nonvital pulps or areas of infection around them, requiring their removal. Therefore, it is important to know the magnitude of the problem followed by treatment planning. ${ }^{10}$

\section{Conclusion}

Retained root fragments carries the risk of future infection or osteoradionecrosis for patients who will receive radiation to the head and neck. The decision to remove retained root fragments either found incidentally on radiographs or those roots that have fractured intra-operatively during tooth extraction procedures needs to be made on a case-by-case basis.

\section{References}

1. Harokopakis-Hajishengallis E. Physiologic root resorption in primary teeth: molecular and histological events. J Oral Sci. 2007;49:1-12.

2. Dachi S F, Howell FV. A survey of 3,874 routine fullmouth radiographs. I. A study of retained roots. Oral Surg Oral Med Oral Pathol 1961;14:916-24.

3. Chung MP, Chen CP, Shieh YS. Floating retained root lesion mimicking apical periodontitis. Oral Surg Oral Med Oral Pathol Oral Radiol Endod 2009;108:63-6.

4. Nairn R I. Interference with function of inferior dental nerve by a root fragment. Oral Surg Oral Med Oral Pathol 1973;36:188-91.

5. Herd J R. The retained tooth root. Aust Dent J 1973;18:125-31.

6. Kharat D U, Saint T. Embedded root fragments in completely and partially edentulous jaws. Saudi Dent J 1991;3:8-12.

7. Bayanzadeh S, Shokri S. Radiographic evaluations of patients seeking removable prostheses treatment. J Dent Med 2004;16:78-81.

8. Ciftci ME, Hastar E, Aktan AM. Evaluation of radiographic features of embedded primary molar roots in adult Turkish population. Niger J Clin Pract 2014;17:7725 .

9. Kramer S. An unexpected adverse reaction due to a retained root. N Y State Dent J 1983;49:768-70.

10. Nyyssönen V, Paunio I, Rajala M. Prevalence of retained roots in the Finnish adult population. Community Dent Oral Epidemiol 1983;11:117-21. 